RAD Conference Proceedings, vol. 2, pp. 198-206, 2017

www.rad-proceedings.org

\title{
THERMAL IMAGING AS A TOOL FOR PATTERN RECOGNITION AND ANOMALY STUDIES: IDENTIFYING THE CHANGES IN THE CONDITION OF AN OBJECT OVER TIME BY SPOTTING A TREND OF CHANGING TEMPERATURES
}

\author{
Gordana Laštovička-Medin*
}

University of Montenegro, Podgorica, Montenegro

\begin{abstract}
More than five decades have passed since the hypothesis of thermography in breast imaging was proposed. During this time, thermography has gone from a legitimate, promising technology to one relegated to the shadows outside conventional medicine. Thermal imaging in clinical trials is still controversial issue. However even those who discard the method due to insufficient reliability of data do not validate their arguments by clear understanding of the reasons behind the inaccuracy. While thermography is not well evidenced for use as a screening tool, its use as an adjunctive imaging procedure alongside mammography should be considered, particularly for those with dense breast tissue. It is certain that images captured by digital infrared thermal imaging support the effective recognition of irregular body patterns and that they can be used as indicators of any anomaly over the time period by spotting a trend of changes in the temperature. But data has to be not only interpreted accurately but also taken carefully and the effect of surrounding environment has to be kept minimal. The identified localized patterns have to be accurately assigned to a certain anomaly in order to be treated as diagnostic method, and the evaluation method as well as interpretation have to be standardized, and method replicable. Moreover, validation of protocols, equipment, and analytical techniques is needed to be placed in the context of large, randomized trials before its use can be considered truly evidence-based. Accurate interpretation of thermal data is largely dependent upon an experienced, knowledgeable operator who understands infrared theory and heat transfer concepts, basic anatomy and physiology, and infrared equipment operation and importantly, limitations too. In this paper we integrate theory behind thermal imaging, potential of thermal imaging in clinical research and general uncertainties and misinterpretations that lead to reduced accuracy of data interpretation and feasibility of the method.
\end{abstract}

Key words: Thermal radiation, infrared radiation, thermal imaging, heat transfer, medical research, energy loss visualization, uncertainties, misinterpretations

DOI: $10.21175 /$ RadProc.2017.41

\section{INTRODUCTION}

The concept of thermal imaging is old. Ring [1] reviewed its application 27 years ago. Considerable progress has been made over last almost 30 years in the performance of infrared equipment, standardization of technique, sensor's calibration and testing of clinical protocols for the case of medical applications. The physiological mechanisms of temperature distributions and electromagnetic waves emitted from body surface are now better understood. The signal processing, theory of information, and miniaturization of electronic components have been subjected to impressive development, too. The significant improvement is done in the resolution in temperature, and focal plane array sensors become common standard enabling better spatial resolution which was needed, for instance, to resolve thermal patterns caused by superficial skin vessels [2].

Smaller camera units and the use of microbolometers lead to higher mobility and imaging of objects in the perpendicular view, i.e. with the camera mounted in the vertical position, which can be now used with modern uncooled equipment. However, for very high sensitive detectors such as the quantum well infrared photodetectors, cooling is still necessary. To address the overall aims, this paper explored the questions

- What are the roles, capabilities and limits of thermal imaging in contemporary science and contemporary digital world? In particular, we researched the role of digital infrared thermal imaging in clinical trials.

The paper is structured around 6 Chapters. Follow the background and context provided in Chapter 1, a more detailed theoretical explanation is given in Chapter 2. The special attention is paid to the concept of emissivity in Chapter 3 while the working principle of thermal imaging camera is briefly explained in Chapter 4. Divisibility of applications is emphasized through Chapters 5 with emphasize on medical applications. The uncertainties involved in 
interpretation of images are given in Chapter 6. Finally, brief conclusion is given in Chapter 7 .

The research method use to prepare this paper combines two approaches - passive and active: analyses of "second hand" data through published papers and eyes of scientists, blogs posted on the internet and through author's active participating in thermal imaging by volunteering in Low Carbon Community and Energy Action Groups in Oxford which enables author to gather own experiences and simulate different environmental conditions to study possible sources of systematic errors.

\section{THERMAL RADIATION}

This chapter provides brief discussion on concepts and laws in thermodynamics which are basics for working principles of thermal imaging technique.

As any atom or molecule inside any object vibrates their average kinetic energy is represented by temperature. Each vibrating atom contains a nucleus and an electronic cloud, which is an orbiting electric charge. According to laws of electrodynamics, a moving electric charge is associated with a variable electric field that produces an alternating magnetic field. In turn, when the magnetic field changes, it results in a coupled with it changing electric field, and so on. Thus, a vibrating particle is a source of electromagnetic field which propagates outwardly with the speed of light and is governed by the laws of optics, that is, the electromagnetic waves can be reflected, filtered, refracted, focused etc.

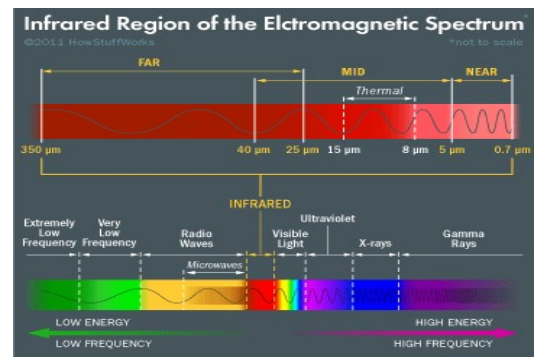

Figure 1. Electromagnetic radiation spectrum [2]

The electromagnetic radiation associated with heat is called thermal radiation. Figure 1 shows the total electromagnetic radiation spectrum covering region from gamma to radio-waves. Thermal radiation is predominantly situated in the mid- and far-infrared (IR) spectral ranges. The wavelength of the infrared radiation 'band' is 0.78 to $1000 \mu \mathrm{m}$ (micrometers).

The wavelength of the radiation, frequency and speed of light $c$ are linked as follows:

$$
\lambda=\frac{c}{v}
$$

However, a relation between $\lambda$ and the temperature is more complex and governed by Planck's law, which was discovered in 1901. It establishes radiant flux density $W \lambda$ as function of a wavelength $\lambda$ and absolute temperature $T$. Radiation flux density as power of electromagnetic radiation per unit of wavelength as follows:

$$
W_{\lambda}=\varepsilon(\lambda) \cdot \Omega_{0} \cdot C_{1} \frac{1}{\pi \lambda^{5}\left(e^{C_{2} / \lambda \cdot T}-1\right)}
$$

where $=\varepsilon(\lambda)$ is the emissivity of the surface from which the electromagnetic field is emitted, $\mathrm{C}\left(=2 \pi h c^{2}\right)_{1}=3.74$ $\mathrm{x} 10^{-12} \mathrm{~W} \mathrm{~cm}{ }^{2}$ is the first radiation constant and $\mathrm{C}_{2}=$ $h c / k=1.44 \mathrm{~cm} \mathrm{~K}$ is the second radiation constant, ' $\Omega_{0}$ is the unit opening angle and $\mathrm{e}$ is the base of natural logarithms [3]. It is important to note that this fundamental equation defines radiation power at a specific wavelength as function of the surface temperature $T$.

Temperature is a result of average kinetic energies of an extremely substantial number of vibrating particles. However, all particles do not vibrate with the same frequency or magnitude. The most probable wavelength is established by Wien's law which can be found by equating to zero the first derivative of equation that describes the Planck's law. The results of calculation are the wavelength near which the most radiation occurs:

$$
\lambda_{m}=\frac{2898}{T}
$$

where $\lambda_{\mathrm{m}}$ is a value given in $\mu \mathrm{m}$ and $T$ in $\mathrm{K}$. Wien's law states that the higher the temperature the shorter is the wavelength. It relates the wavelength at which the maximum power is emitted by a black body to its temperature. A black body is an idealized physical body that absorbs all incident electromagnetic radiation, regardless of frequency or angle of incidence. A black body in thermal equilibrium emits electromagnetic radiation called black-body radiation. The radiation is emitted according to Planck's law, meaning that it has a spectrum that is determined by the temperature alone, not by the body's shape or composition

However, in practice it may be difficult to determine $\lambda$ precisely because:

- The instrument used to detect the radiation may not have the same sensitivity for all wavelengths;

- The atmosphere absorbs some wavelength of light more than others, so the intensity of these wavelengths may be reduced by more than that of others as the electromagnetic waves travels through the atmosphere. This is very important in physics of stars and in the Earth satellite monitoring.

To sum up above explained: the radiate frequency and the wavelength depend only on temperature of object, while the magnitude of radiation also depends on the emissivity of the surface.

Figure 2 shows the radiant flux density for a set of different temperatures. It is seen that the radiant energy is distributed over the spectral range highly non-uniformly, with a clearly pronounce the maximum defined by the Wien's law. The dotted line in Figure 2 (right) shows how the wavelength at which the maximum power is emitted depends on the temperature of the black body. 

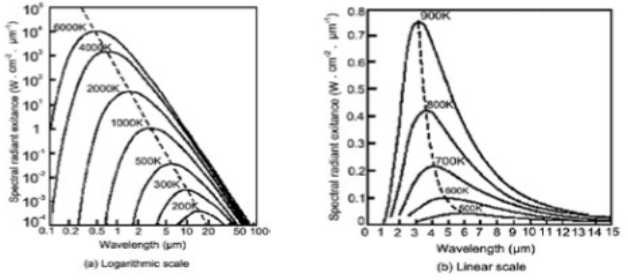

Figure 2. The radiant flux density for a set of different temperatures

Theoretically, a thermal radiation bandwidth is infinitely wide. However, when detecting radiation, limited properties of hardware - real sensors have to be taken into account. Each sensor is sensitive a specific range of wavelength band. Thus, in order to determine the total radiated power within a particular bandwidth, equation which presents Planck's law is integrated within wavelength range limit. A solution of integrated Planck's equation is complex and cannot be obtain analytically. A solution can be found either numerically or by an approximation. A useful approximation when range of wavelength cover at least $50 \%$ of the total radiated power is known as the Stefan-Boltzmann law:

$$
\phi=A \cdot \varepsilon \cdot \sigma \cdot T^{4}
$$

where variable $\sigma=5.67 \times 10^{-8} \mathrm{~W} / \mathrm{m}^{2} \mathrm{~K} 4$ (Stefan Boltzmann constant), A is the geometry factor, and emissivity $\varepsilon$ is assumed to be wavelength independent. In reality, any surface that faces another surface of its own temperature absorbs as well from them their thermal flux. This concept is fundamental in developing thermal sensors. The concepts such as emissivity, transparency and reflectivity will be explained in the Chapter 3 .

\section{EMISSIVITY}

The concept of emissivity is very important for the accurate interpretation of the thermal images. Emissivity is measured on a scale from o to 1. It is ratio of the actual radiated flux to that which would be radiated from an ideal emitter, having the same temperature.

When thermal radiation flux (determined by Stefan - Boltzmann law) is detected by thermal sensor having its own temperature, the opposite flowing radiation from sensor toward the observed object must also be accounted for. However, a thermal sensor is only capable of responding only to a net formal flux, for example, flux from the object towards sensor $\Phi_{1}$ minus flux from the sensor towards object $\Phi_{2}, \Phi_{1}-\Phi_{2}$ (see Figure 3). The sensor's surface has emissivity, and reflectivity, and hence the sensor is only partially absorptive, this means that not the entire flux, $\Phi_{1}$, is absorbed, and therefore utilized. This means that only fraction of $\Phi, \eta \Phi_{1}$ is absorbed. Another part, $\Phi_{\text {lost }}=1$ $\eta \Phi_{1}$, is reflected towards the monitored object and lost. The reflectivity flux is proportional to the sensor's coefficient of reflectivity:

$$
\phi_{\text {lost }}=-\rho_{s} \phi_{1} \text {. }
$$

The net flux originating by the object and utilize by the sensor depend on both, the object's and the

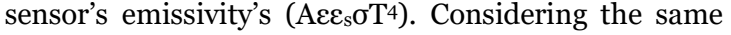
principle but this time applying it on the sensor (see illustration of thermal radiation exchange between the object and the sensor) the mathematical model, on which the net thermal flux is based, is as follows:

$$
\phi=A \cdot \varepsilon \cdot \varepsilon_{s} \cdot \sigma\left(T^{4}-T_{s}^{4}\right) .
$$

To conclude, when energy loss is monitored, it is important to understand the optical features of surrounding material. This is particularly important when survey presents indoor inspection since surrounding objects such a mirror, white paper, pictures can affect the validity of captured images. For example, white sheet paper is very much reflective in the visible spectral range and emits no visible light. In the mid- and far-infrared spectral ranges its reflectivity is low and emissivity is high (about 0,92). Therefore, white paper presents good emitter of thermal radiation.

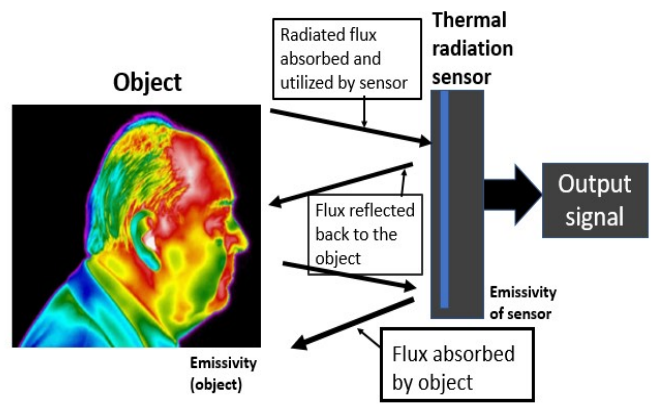

Figure 3. Thermal radiation exchange between object and thermal radiation sensor

\section{IMAGING CAMERA: WORKING PRINCIPLES}

The working concept of the thermal imaging camera is as follows: infrared energy coming from an object is focused by the optics onto an infrared detector. The detector sends the information to the sensor electronics for image processing. The electronics translate the data coming from the detector into an image that can be viewed on the viewfinder or on a standard video monitor or LCD screen.

Infrared thermography is the art of transforming an infrared image into a radiometric one, which allows temperature values to be read from the image. Every pixel in the radiometric image is in fact a temperature measurement. In order to do this, complex algorithm is incorporated in the thermal image camera. Representation of picture is given by false colors - in order to emphasize better visibility, the recorded data are mapped using different colors that represent the different intensities of emitted radiation.

The temperature shown on images does not always correspond to the actual one. As emphasized in [26] false colour coding of infrared images does not provide means for temperature measurement. If colours are separated by a temperature distance of $1^{\circ} \mathrm{C}$, the temperature difference between two points situated in 
adjacent colours may be between 0.1 and $1.9^{\circ} \mathrm{C}$. It is obvious, that false coloured images provide at its best an estimation of temperature, but not a measurement. The same is true for liquid crystal thermograms. Furthermore, Ammer and Ring in [26] clearly explained that temperature measurements in thermal images are based on the definition of regions of interest (ROI) and that standards for shape, size and placement of these regions are not available or incomplete. Although a close correlation exists for ROI of different size in the same region, the precision of measurement is affected when ROIs of different size and location are used for repeated measurements. The field of view has to be defined in the standard protocol Spatial resolution depends on distance to the inspected object and the best option has to be optimized taking into account the number of pixels and temperature resolution. Articles that are referred in [26] offer more details in this subject. Much more about uncertainties and some estimations will be given in Chapter 6 .

\section{APPLICATIONS}

Unlike images created by $\mathrm{x}$-rays or proton activation through magnetic resonance, thermal imaging is not related to morphology. The technique provides only a map of the distribution of temperatures on the surface of the object imaged. In 1979 it was proved that the human body and tissues emanate weak electromagnetic waves [4]. However, early research did not favor Digital Infrared Thermal Imaging (DITI) in clinical trials [5-7].

However, a recently reawaken trend of growing divisibility and applicability of thermal imaging in medical diagnostics of breast abnormalities, thyroid abnormalities, musculoskeletal, peripheral vascular [8], diabetes [9], cerebral vascular, inflammatory and neoplastic conditions) is observed. The reason to use thermal imaging technology in medical diagnostics is because temperature is a very good indicator of health, as changes of just a few degrees on the skin (cutaneous or superficial) can be used as indicator of possible illnesses [10], Fig 4. The spectrum of colours indicate an increase or decrease in the amount of infrared radiation being emitted from the body surface. Since there is a high degree of thermal symmetry in the normal body, subtle abnormal temperature asymmetry's can be easily identified.
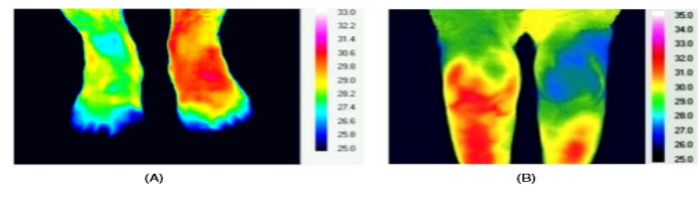

Figure 4. Chronic inflammation of the forefoot following a sports injury (a); rheumatoid arthritis of one knee (b) [10]

Digital thermal imaging was used to study an emotion-related disorder such as schizophrenia too [11]. Schizophrenia is a neurological disease characterized by alterations to patients' cognitive functions and emotional expressions. Relevant studies often use magnetic resonance imaging (MRI) of the brain to explore structural differences and responsiveness within brain regions. However, as this technique is expensive and commonly induces claustrophobia, it is frequently refused by patients. Thus, as novel approach which is less expensive and non-invasive, authors in [11] used non-contact infrared thermal facial images (ITFIs) to analyze facial temperature changes evoked by different emotions in moderately and markedly ill schizophrenia patients. The average temperatures from the forehead, nose, mouth, left cheek, and right cheek were calculated, and continuous temperature changes were used as features. After performing dimensionality reduction and noise removal using the component analysis method, multivariate analysis of variance and the Support Vector Machine (SVM) classification algorithm were used to identify moderately and markedly ill schizophrenia.

Furthermore, thermal imaging technique may be of great value to provide early and reliable burn wound assessment. Current practices, however, often overlook the importance of emissivity when taking thermal measurements. The consensus is that human skin has an emissivity of 0.98 but this value varies between individuals, areas examined, and if the skin is damaged. Further, research should be conducted on the emissivity variations of wounds [12].

Here we will focus our attention on the breast thermography. Particularly the advanced programming needed to analyse images will be explored. Breast thermography is a physiologic test measuring subtle differences in skin temperature that can be associated with an underlying tumour (as well as other pathology). It records the temperature distribution of a body using the infrared radiation emitted by the surface of that body. Precancerous tissue and the area around a cancerous tumour have higher temperature due to angiogenesis, and higher chemical and blood vessel activity than a normal breast; hence breast thermography has potential to detect early abnormal changes in breast tissues. It can detect the first sign of forming up cancer before mammography can detect. The thermal information can be shown in a pseudo coloured image where each colour represents a specific range of temperature. Various methods and techniques can be applied to extract hot regions for detecting suspected regions of interests (ROI) in the breast infrared images and potentially suspicious tissues such as image segmentation techniques [13], feature extraction techniques [14] where the ability of feature set in differentiating abnormal from normal tissue is investigated using a Support Vector Machine classifier, Naive Bayes classifier and K-Nearest Neighbour classifier. Features extraction and selection aims to derive descriptive characteristics of the extracted object, which are similar within the same object class and different between different objects. This will facilitate the last step of the image analysis process: pattern classification. The goal of pattern classification is to assign a class to the selected features from a group of known classes. There are two types of classifier learning algorithms: supervised and unsupervised. Supervised learning algorithms predict the class of the object under test using training data of known classes. The training data have a predefined label for every class and the learning algorithm can utilize this data to predict the class of a test object. Unsupervised learning 
algorithms use unlabelled training data and divide them into groups using similarity measurements. Unsupervised learning algorithms predict the group to which a new test object belong to, based on the training data without giving an explicit class to that object. Maximum likelihood techniques have had some success, but are very susceptible to the choice of training region. A robust segmentation technique based on an extension to the traditional fuzzy c-means (FCM) clustering algorithm is proposed in [15]. A neighbourhood attraction, which is dependent on the relative location and features of neighbouring pixels, is shown to improve the segmentation performance dramatically. The degree of attraction is optimized by a neural-network model. The discrete wavelet transform (DWT) and texture features extracted from thermograms in classifying normal and abnormal groups were investigated in $[16 \mid$. Asymmetry analysis of texture features was used in [17|. The texture features are extracted from the breast region and fed to a back propagation neural network for automatic detection of abnormal breast thermograms. The classifier is able to classify abnormal and normal thermograms with an accuracy of $85.19 \%$. Lisboa in [18] paper reports on a systematic review that was conducted to assess the benefit of artificial neural networks (ANNs) as decision making tools in the field of cancer. The $3 \mathrm{D}$ computational model of the breast with COMSOL FEM software was used in [19] where the effect of various parameters (tumour size, location, metabolic heat generation and blood perfusion rate) on the surface temperature distribution (which can be measured with infrared thermography) has been analysed. Key defining features of the surface temperature profile have been identified, which can be used to estimate the size and location of the tumour based on (measured) surface temperature data. In addition, authors employed a dynamic cooling process, to analyse surface temperature distributions during cooling and thermal recovery as a function of time and successfully demonstrated that a quantification of temperature distributions by computational modelling, combined with thermographic imaging and dynamic cooling can be an important tool in the early detection of breast cancer.

In what follows we will compare mammography to thermal imaging. Breast cancer is the most common malignancy in women. Screen-film mammography (SFM) has been considered the gold standard for breast cancer screening and detection. Despite its recognized value in detecting and characterizing breast disease, mammography has important limitations and its false-negative rate ranges from $4 \%$ to $34 \%$. Given these limitations, development of imaging modalities that would enhance, complement, or replace mammography has been a priority. Digital mammography (FFDM) and digital infrared thermal imaging (DITI) are some of these alternative modalities explored and compared in [20]. Furthermore, female breast tissue is highly sensitive to radiation, and this electromagnetic radiation triggers the factor that is responsible for cancerous growth. The radiation also raises the possibility of spreading or metastasizing an existing growth [21,22] However, in case of dense breast tissue in younger women, detection of suspicious lesions is very difficult from mammography.
Compare to the older women of age above 50, breast cancers grow very faster in younger women under 40 years. The faster a malignant tumor grows the amount of infrared radiation it generates is also greater that can be recorded using a very sensitive thermal camera. Gautherie et al. suggested that an abnormal thermogram is the single most reliable indicator of high risk of breast cancer in its early stage [23]. There is many more studies and all cannot be included, so we will summarize the common findings: the clinical uses for DITI include the following:

- To define the extent of a lesion of which a diagnosis has previously been made;

- To localize an abnormal area not previously identified, so further diagnostic tests can be performed;

- To detect early lesions before they are clinically evident.

When we judge methods or techniques and giving preferences in chooses we have to keep in mind that there is no one test that can detect $99-100 \%$ of all cancers. Cross check and cross overlapping of images are important for safety and security of patients. Therefore, no single test exists that can be used alone as an adequate screening or detection method for breast cancer. A physiological imaging procedure (thermography) cannot replace an anatomical imaging procedure (mammography) but can advance cancer research and offer additional information. However, it is of crucial importance to understand that the two tests are "looking" for completely different pathological processes and thus should not compete against each other but researchers should support each other and look for innovative and maybe unconventional resources to understand better the cancer behavior and response of human body to it.

Another application of thermal imaging in clinical studies is Thermal Texture Maps in SARS Diagnosis [24]. There are many more papers which reported results of thermal imaging used in clinical studies. However, it is very difficult to judge reliability of data and claim benefits or disprove the methods of thermal imaging in cancer research due to lack of larger statistics and due to a variety of thermal imaging cameras and a variety of software used for data analyses. Also, different protocols are applied. Some rewires emphasizes the benefits of use thermal imaging in cancer research while others are concern with poor reliability of methods [25]. The Thermal Imaging in clinical trials is questioned by many authors but also supported by others. However, one common thing is clear: there is no sufficient data which would significantly support the accuracy of method since there is visible lack of conducted research within EU towards the understanding the inaccuracy of data and sources of uncertainty. In the next Chapter, we will focus our attention on general uncertainties involved with thermal imaging technique that are also applicable in medical research.

\section{GENERAL UNCERTAINTIES AND MISINTERPRETATIONS}

Measurements are basic procedures of comparison namely to compare a standardized meter with an object 
to be measured. Any measurement is prone to error; thus, a perfect measurement is impossible. However, the smaller the variation of a measurement from the standardized meter, the higher is the accuracy of the measurement or in other words, an accurate measurement is as close as possible to the true value of measurement. Whenever infrared thermal imaging is considered as a method for measurement, the technique must meet all criteria of a measurement. The most basic features of measurement are accuracy (in the medical field also named validity) and precision (in medicine reliability) [26]. In medicine, accuracy is often named validity, mainly caused by the fact, that medical measurements are not often performed by the simple comparison of meter and object. For example, assessments from various features of a human being may be combined into a new construct, resulting in a innovative measurement of health [31]. The smaller the variation between single results, the higher is the precision or repeatability (reliability) of the measurement. However, reliability without accuracy, is useless.

Conditions affecting the accuracy, precision and responsiveness of temperature measures are as follows: object or subject, camera systems, standards, and calibration, angle viewing - patient position and image capture, information protocols and resources, image analysis, image exchange, image presentation, knowledge and skills of examiner, software which used.

Both, accuracy and precision, have an impact on the sensitivity to change of outcome measures. Validity is needed to define correctly the symptom to be measured. Precision will affect the responsiveness also, because a change of the symptom can only be detected if this change is bigger than the variation of repeated measurement.

Identification, characterization and verification of uncertainties when detecting the loss of heat due to faulty insulation are complex procedures. The possible errors assigned to a measurement system itself is the following: calibration of camera sensitivity and systematic offsets in sensor, conversion of apparent to true temperature, camera optics, electronic effects, instrument error from instrument noise, integrated averaging of radiance over increasing pixel area due to increased viewing distance (decreased resolution) etc. Other uncertainty sources are linked to ambient conditions such as weather humidity, atmospheric pressure and ambient temperature. For example, infrared sensed data can be subjected to errors greatly due to atmospheric attenuation by atmospheric scattering caused by particulate material in the atmosphere and absorption by gases.

The errors arising from viewing the surface at an oblique angle can significantly affect the measurement accuracy and thus the validity of data/image interpretation. The uncertainty in calibration of camera, sensitivity and offset may be linked to incorrect adjustment of camera to emissivity of inspected object. Furthermore, the dependency of camera sensitivity towards polarization of the measured thermal radiation brings uncertainty as well. So, to improve accuracy the camera system must vary as a function of polarization angle. Also, an incomplete understanding of resolution (the smallest possible distance between two values of measurement) and repeatability (the range of values attained by repeated measurements under the same conditionals) may be a significant source of uncertainty.

Other problems associated with the infrared thermograph are the surrounding radiation reflected by the target surface and the atmospheric absorption of radiation. The infrared thermography test applied to a liquid lead cooling process is a clear example of the advantages and disadvantages of non-contact temperature measurements using infrared techniques. Distance target-camera, monitoring temperature within a scene simultaneously, visibility, fast response rate, portability and data manipulation are highlighted advantages applied to this application. But, the target to be tested has the following characteristics: it is nonplanar surface and its emissivity could change during the process, which are grave problems in applying thermography. Also, the measured radiation maybe influenced by the presence of flames between the camera and the target and produced by the heating process. Beside conduction and convection, the surface area to volume ratio also influences all aspects of heat exchange particularly in the case of animal (high ratio increases rates of heat exchange).

Hardy showed that the human skin acts like an almost perfect black body radiator with an emissivity of 0.98 [27]. Studies from Togawa in Japan have demonstrated that the emissivity of the skin is unevenly distributed [28]. In addition, infrared reflection from the environment and substances applied on the skin may also alter the emissivity [29, 30].

Here we will discuss the error originating from not knowing the emissivity of an object. The radiometric thermographs are calibrated on black body. Thus, when the object with much lower emissivity then 1 is inspected, the measurement result is subjected to the larger error. Spectral radiation at a specific wavelength originating from the object with emissivity $\varepsilon<1$ at a specific temperature $\mathrm{T}_{0}$ is given by the following term

$$
\mathrm{Wo}(\lambda)=\varepsilon \operatorname{c1e}(-\mathrm{c} 2 / \lambda-\mathrm{To}) /\left(\pi^{\prime} \Omega 0 \lambda_{5}\right)
$$

However, the infrared (IR) thermometer will show the different value, Tc, corresponding to the black body which would have the same radiation density as inspected body.

$$
\mathrm{Wb}(\lambda)=\varepsilon \mathrm{c1e}(-\mathrm{c} 2 / \lambda-\mathrm{Tc}) /\left(\pi^{\prime} \Omega 0 \lambda_{5}\right)
$$

After applying $\mathrm{W}_{\mathrm{o}}(\lambda)=\mathrm{W}_{\mathrm{b}}(\lambda)$, the difference between actual and measured temperature is calculated:

$$
1 / \mathrm{To}-1 / \mathrm{Tc}=\lambda / \mathrm{c} 2 \ln (\varepsilon)
$$

Thus, their temperature which is read by radiometer is always lower than actual one. However, error due to assumed object's emissivity is lower than error in reading the temperature because the emissivity is parameter of logarithmic function. For instance, if actual object's emissivity is 0.6 , and with IR thermometer is the measured temperature $\mathrm{Tc}=1273$ $\mathrm{K}$, this means that actual temperature of the object is $1311 \mathrm{~K}$. So, the error due to assumption that emissivity of object is 1 and not 0.6 brings error of only 3\% while error in emissivity is $40 \%$. IR thermometers with 
sensors sensitive to heat such as bolometers measure the net radiation heat where the spectral density is defined by Stephan- Boltzmann law. The radiation from object with emissivity $\varepsilon$ and coefficient of reflection $\rho$ contains two parts: the first part originates from radiation of object itself and is equal to $\Phi_{1}=\varepsilon \sigma \mathrm{T} 4$ while second part originates from ambient (surrounding - sensor and medium such as air) and is reflected from the object's surface; it is equal to $\Phi_{2}=$ $\rho \sigma \mathrm{T}_{1} 4=(1-\varepsilon) \mathrm{T}_{1} 4$ where value $\mathrm{T}_{1}$ is the temperature of surrounding (ambient temperature). Here it is assumed that surrounding behave as a black body $(\varepsilon=1)$. The net spectral density is described by following term

$$
\Phi=\varepsilon \sigma \mathrm{T} 4+\rho \sigma \mathrm{T} 14=(1-\varepsilon) \sigma \mathrm{T} 14=\sigma \mathrm{TC} 4,(10)
$$

where $\mathrm{T}_{\mathrm{c}}$ corresponds to the equivalent temperature of black body shown by thermometer. The actual temperature is then obtained as follows:

$$
T=\frac{T_{c}}{\sqrt[4]{\varepsilon}}\left[1-(1-\varepsilon)\left(\frac{T_{1}}{T_{c}}\right)^{4}\right]^{1 / 4}
$$

If the ambient temperature $\mathrm{T}_{1}$ is much lower than measured temperature $\mathrm{T}_{\mathrm{c}}$, the previous equation becomes:

$$
T=T_{c}(\varepsilon)^{-1 / 4}
$$

As an example, we will calculate error assuming that actual object's emissivity is 0.6 and that the recorded temperature is $\mathrm{T}_{\mathrm{c}}=1273 \mathrm{~K}$. Using equation (2) as actual temperature we obtain $\mathrm{T}=1446 \mathrm{~K}$. This means that error is $12 \%$, what is lower than the error caused by assuming body to have emissivity as black body $(\varepsilon=1)$.

\section{CONCLUSION}

To conclude the main advantages of infrared thermographs (thermal imaging technique) are the following: IRT is a noncontact technology. This means that devices are not in contact with the source of heat, i.e., they are non-contact thermometers; they provides two-dimensional thermal images, which make a comparison between areas of the target possible, imaging is in real time, which enables not only highspeed scanning of stationary targets, but also acquisition from fast-moving targets and from fast changing thermal patterns; it has none of the harmful radiation effects of technologies, such as X-ray imaging and thus, it is suitable for prolonged and repeated use; thermal imaging is a non-invasive technique, thus, it does not intrude upon the target or affect it in any way and it can also be easily incorporated into neurological and psychological studies or work with children with behavior disorders and neurological diversity.

However, thermal imaging camera and infrared thermograph are not without drawbacks. Fast and affordable hardware has recently become available, but an infrared camera is still an expensive device. Other, inexpensive models with high spatial resolution provide lower accuracy, which makes them unusable for some applications. Infrared images can also be difficult to interpret; in general, specific training is required. Infrared imaging is also highly dependent on working conditions, such as the surrounding temperature, airflow or humidity. Thus, progress on accuracy of image interpretation in needed. In order to make thermal imaging a universally acceptable clinical technique, one must try to understand what is being observed, and what the observation signifies. One must also prove the validity of hypotheses about underlying causes of a given local hyper or hypothermia. An interesting study was done on healthy people in order to evaluate reliability and reproducibility of images. Their results suggest that the IRT technique may represent an objective quantifiable indicator of autonomic disturbances although there are considerable temporal variations in the measured values which are due to both technical factors such as equipment accuracy, measurement environment and technique, and physiological variability of the blood flow, and these factors should be taken into account.

Regarding screenings, the point is that screening tests, though important, are imperfect. It may make sense to have them, but it doesn't make sense to rely on them completely. The medical research is not the final word on reality. Like medicine itself, it's as much an art as it is a science, and the science is based largely on statistics. Relying too heavily on statistics is dangerous because they can be mathematically correct and grossly misleading at the same time. Research approximates of reality. It is not reality itself. Reality has too many variables. No research study can control them all. But can improve our limits in understanding the limits of technology. Further investigations can help us to understand the boundaries of method imposed by uncertainties and remove them if possible by bringing a new approaches and more advanced software and electronics.

Acknowledgement: The paper is a part of the research supported by Montenegro's Ministry of Science and Ministry of Education.

\section{REFERENCES}

1. E. F. J. Ring, "Quantitative thermal images," Clin. Phys. Physiol. Meas., vol. 11, no. suppl. A, pp. 87 - 95, 1990. DOI: $10.1088 / 0143-0815 / 11 / 4 \mathrm{~A} / 310$ PMID: 2286052

2. E. F. J. Ring and J. M. Dicks, "Spatial resolution of new thermal imaging systems," Thermol. Int. vol. 9, no. 1, pp. 7- 14, 1999.

3. J. Fraden, Handbook of modern sensors, Physics, design and applications, 5th ed., San Diego (CA), USA: Springer, 2016. DOI: $10.1007 / 978-3-319-19303-8$

4. R. Dobrin, C. Kirsch, S. Kirsch et al., "Experimental measurements of the human energy field," Psychoenergetic Systems, vol. 2, pp. 213 - 216, 1979.

5. C. R. Hitchcock, D. F. Hickok, J. Soucheray, T. Moulton, R. C. Baker, "Thermography in mass screening for occult breast cancer," JAMA. vol. 204 no. 6, pp. $419-422$, May 1968.

DOI: 10.1001/jama.1968.03140190001001 PMid: 5694429

6. S. A. Feig, G. S. Shaber, G. F. Schwartz et al., "Thermography, mammography, and clinical examination in breast cancer screening: Review of 
16,000 studies," Radiology, vol. 122, no. 1, pp. 123 127, Jan. 1977.

DOI: $10.1148 / 122.1 .123$

PMid: 830320

7. M. Kontos, R. Wilson, I. Fentiman, "Digital infrared thermal imaging (DITI) of breast lesions: sensitivity and specificity of detection of primary breast cancers," Clin. Radiol., vol. 66, no. 6, pp. 536 - 539, Jun. 2011.

DOI: 10.1016/j.crad.2011.01.009

PMid: 21377664

8. S. Bagavathiappan, T. Saravanan, "Infrared thermal imaging for detection of peripheral vascular disorders," J. Med. Phys., vol. 34, no. 1, pp. 43 - 47, Jan. 2009.

DOI: $10.4103 / 0971-6203.48720$

PMid: 20126565 PMCid: PMC2804148

9. F. Ring, "Thermal imaging today and its relevance to diabetes", J. Diabetes Sci. Technol., vol. 4, no. 4, pp. 857 - 862, Jul. 2010.

DOI: $10.1177 / 193229681000400414$

PMid: 20663449

PMCid: PMC2909517

10. E. F. J. Ring and K. Ammer, "Infrared thermal imaging in medicine," Physiological Measurement, vol. 33, no. 3, pp. 33- 46, Feb. 2012.

DOI: $10.1088 / 0967-3334 / 33 / 3 / \mathrm{R} 33$

PMid: 22370242

11. B. L. Jian, C. L. Chen, W. L. Chu, M. W. Huang, "The facial expression of schizophrenic patients applied with infrared thermal facial image sequence," $B M C$ Psychiatry., vol. 17, no. 1, p. 229, Jun. 2017.

DOI: $10.1186 / \mathrm{s} 12888-017-1387-\mathrm{y}$

PMid: 28646852 PMCid: PMC5483292

12. E. Keenan, G. Gethin, L. Flynn, D. Watterson, G. M. O'Connor, "Enhanced thermal imaging of wound tissue for better clinical decision making," Physiol. Meas., vol. 38, no. 6, pp. 1104-1115, May 2017.

DOI: $10.1088 / 1361-6579 /$ aa6eao

PMid: 28430667

13. N. Golestani, M. EtehadTavakol, E. Ng, "Level set method for segmentation of infrared breast thermograms," EXCLI J., vol. 13, no. 13, pp. 241 - 251, Mar. 2014.

PMid: 26417258

PMCid: PMC4464455

14. M. Milosevic, D. Jankovic, A. Peulic, "Thermography based breast cancer detection using texture features and minimum variance quantization," EXCLI J., vol. 13, pp. $1204-1215$, Nov. 2014.

PMid: 26417334 PMCid: PMC4464488

15. S. Shen, W. Sandham, M. Granat, A. Sterr, "MRI fuzzy segmentation of brain tissue using neighbourhood attraction with neural-network optimization," IEEE Trans. Inf. Technol. Biomed., vol. 9, no. 3, pp. 459 467, Sep. 2005.

DOI: 10.1109/TITB.2005.847500 PMid: 16167700

16. M. R. K. Mookiah, U. R. Acharya, E.Y. K. Ng, "Data mining technique for breast cancer detection in thermograms using hybrid feature extraction strategy," J. Quantit. IR Thermography, vol. 9, no. 2, pp. $151-$ 165, Nov. 2012.

DOI: $10.1080 / 17686733.2012 .738788$

17. S. V. Francis, M. Sasikala, "Automatic detection of abnormal breast thermograms using asymmetry analysis of texture features," J. Med. Eng. Technol., vol. 37, no. 1, pp. $17-21$, Nov. 2012.

DOI: $10.3109 / 03091902.2012 .728674$ PMid: 23194447

18. P. J. Lisboa, A. F. Taktak, "The use of artificial neural networks in decision support in cancer: a systematic review," Neural. Netw., vol. 19, no. 4, pp. $408-415$, May 2006

DOI: 10.1016/j.neunet.2005.10.007

PMid: 16483741

19. A. Chanmugam, R. Hatwar, C. Herman, "Thermal analysis of cancerous breast model," in Proc. Int. Mech. Eng. Congress Expo., 2012, pp. 134 - 143.

DOI: 10.1115/IMECE2012-88244

PMid: 25328914 PMCid: PMC4199207

20. N. Kösüs, A. Kösüs, M. Duran, S. Simavll, N. Turhan, "Comparison of standard mammography with digital mammography and digital infrared thermal imaging for breast cancer screening," J. Turk. Ger. Gynecol. Assoc., vol. 11, no. 3, pp. $152-157$, Sep. 2010.

DOI: $10.5152 /$ jtgga.2010.24

PMid: 24591923 PMCid: PMC3939224

21. S. H. Kobrunner, A. Hacker, S. Sedlacek, "Advantages and disadvantages of mammography screening," Breast Care (Basel, Switzerland), vol. 6, no. 3, pp. $199-207$, Jun. 2011.

DOI: $10.1159 / 000329005$

PMCid: PMC 3132967

22. D. Kennedy, T. Lee, D. Seely, "A comparative review of thermography as a breast screening technique," Integr. Cancer Ther., vol. 8, no. 1, pp. 9-16, Feb. 2009.

DOI: $10.1177 / 1534735408326171$ PMid: 19223370

23. M. Gautherie, C. M. Gros, "Breast thermography and cancer risk prediction," Cancer, vol. 45, no. 1, pp. 51 56, Jan. 1980.

DOI: $10.1002 /$ cncr.2820450110 PMid: 7351006

24. W. Wang, Y. Zeng et al., "Clinical Study on Using Thermal Texture Maps in SARS Diagnose," in Proc. 26th Annual International Conference of the IEEE EMBS, San Francisco (CA), USA, 2004, pp. 5258 5264 .

DOI: 10.1109/IEMBS.2004.1404469

25. M. Norzakiah, Thermography: infrared thermal imaging: Technology review, S. Sadasivan, Ed., Putrajaya, Malaysia: Health Technology Assessment Unit, Ministry of Health Malaysia, 2005.

Retrieved from:

http://www.moh.gov.my/index.php/database stores/a ttach download/347/39

Retrieved on: Jan. 20, 2017

26. K. Ammer, E. F. J. Ring, "Standard Procedures for Infrared Imaging in Medicine," in Medical Infrared Imaging: Principles and Practice, M. Diakides, J. D. Bronzino, D. R. Peterson, Eds., Boca Raton (FL), USA: CRC Press, 2013, ch. 32. pp. $32.1-32.14$. Retrieved from:

https://www.researchgate.net/publication/233986901 Standard Procedures_For_Infrared_Imaging in $M$ $\underline{\text { edicine }}$

Retrieved on: Jan. 20, 2017

27. J. D. Hardy, "The radiation of heat from the human body. III. The human skin as a black body radiator," J. Clin. Invest., vol. 13, pp. 615 - 620, Jul. 1934. DOI: 10.1172/JCI100609

28. T. Togawa, H. Saito, "Non-contact imaging of thermal properties of the skin," Physiol. Meas., vol. 15, no. 3, pp. $291-298$, Aug. 1994. DOI: $10.1088 / 0967-3334 / 15 / 3 / 007$ PMid: 7994207

29. J. M. Engel, "Physical and physiological influence of medical ointments of infrared thermography," in Recent Advances in Medical Thermology, E. F. J. Ring, B. Phillips, Eds., New York (NY), USA: Plenum Press, 1984, pp. $177-183$.

DOI: $10.1007 / 978-1-4684-7697-2$ 
30. S. Hejazi, M. Anbar, "Effects of topical skin treatment and of ambient light in infrared thermal images," Biomed. Thermol., vol. 12, pp. 300 - 305, Jan. 1993.

31. N. Zaproudina, V. Varmavuo, O. Airaksinen and M. Närhi, "Reproducibility of infrared thermography measurements in healthy individuals," Physiological Measurement, vol. 29, no. 4, pp. 515 - 524, Apr. 2008. DOI: 10.1088/0967-3334/29/4/007

PMid: 18401069 